\section{QSAR models and their practi- cal use. The experience of Kode}

\author{
Alberto Manganaro \\ Kode Chemoinformatics s.r.l., Pisa, Italy
}

\begin{abstract}
Kode s.r.l. is a consultancy company founded in 2012, since the beginning having a department focused on the chemoinformatics topics (that became, in 2019, a new company named Kode Chemoinformatics s.r.l., subsidiary of Kode). Throughout these years, we have been involved in several projects that led to the development of software tools for QSAR, in particular aimed to eco-toxicological assessment. We will briefly show some of these tools and their practical value based on our experience. There is a strong need and request for tools that can be used for quick, reliable and nonexpensive screening and assessment of chemicals, in particular for regulatory activities. In the experience of these past years, we have been able to work on several tools that met a positive feedback from users, and that are actually used both by industries and regulatory bodies.
\end{abstract}

\section{Introduction}

Kode s.r.l. is a consultancy company founded in 2012, since the beginning having a department focused on the chemoinformatics topics (that became, in 2019, a new company named Kode Chemoinformatics s.r.l., subsidiary of Kode). Throughout these years, we have been involved in several projects that led to the development of software tools for QSAR, in particular aimed to eco-toxicological assessment. We will briefly show some of these tools and their practical value based on our experience.

\section{Materials and Methods}

Kode had several projects focused on the development of QSAR models and their implementation in practical tools, some of them freely available for end users (toxicologists, chemists etc.) as the result of publicly funded European projects. We would like to mention some of these projects and the resulting tools.

Concerning the European funded projects, Kode is currently beneficiary in two projects, LIFE VERMEER (LIFE16
ENV/IT/000167; https://www.life-vermeer.eu/) and LIFE CONCERT REACH (LIFE17 GIE/IT/000461). The above mentioned project, together with other EU projects in which Kode has been sub-contractor (Prosil, Caleidos, Combase, EuToxRisk), led to the development of the freely available software platform VEGA (https://www.vegahub.eu/). This platform is currently a stand-alone application, developed in Java, that makes available a wide set of QSAR models. Kode has been involved in different commissions for the German UBA (environmental protection agency) mainly focused on the development of tools needed to screen and evaluate large sets of chemicals for their potential eco-toxicological impact. A first project led to the development of a tool named Prometheus, in which a battery of QSAR models are used to provide PBT (persistent, bio-accumulative and toxic) assessment on large sets of chemicals.

\section{Results}

The participation in many projects led to the development of several tools, that have the practical goal of being used by toxicologists and chemists, who don't have necessarily deep knowledge of the in-silico and QSAR/QSPR topics. In particular, the tools are developed with the main goal of being used for the evaluation and registration under the REACH European legal framework, as a part of a non-testing strategy involving other in-silico tools and approaches (like read-across assessment). ${ }^{1}$

The VEGA platform currently makes available over 50 QSAR models. They are grouped into models for (human) toxicity (like carcinogenicity, reproductive toxicity etc.), eco-toxicity (mainly aquatic toxicity), environmental fate (BCF, persistence etc.) and properties (LogP, water solubility). It just requires as input the structure of the target molecules (as SMILES string or SDF files) and then process the selected models, providing both a text output (easy to be used and imported in spreadsheets and any other software tool) and a complete PDF report.

VEGA is widely used, and also mentioned in an official guide from ECHA, ${ }^{2}$ as one of the in-silico tools that can be used in $\mathrm{REACH}$ registration of chemicals. Indeed, it is actually used as a non-testing method for the assessment of commercial chemicals. Different projects with the German UBA led to the development of Prometheus, ${ }^{3}$ and of its extension Janus (the project is still ongoing and the final application is not released yet). Prometheus (and in
Correspondence: Alberto Manganaro, Kode Chemoinformatics s.r.l., Pisa, Italy.

E-mail: a.manganaro@kode-solutions.net

Key words: QSAR; chemoinformatics; ecotoxicology.

Conference presentation: this paper was presented at the Second Centro 3R Annual Meeting - 3Rs in Italian Universities, 2019, June 20-21, University of Genoa, Italy.

Received for publication: 28 October 2019. Accepted for publication: 6 November 2019.

This work is licensed under a Creative Commons Attribution NonCommercial 4.0 License (CC BY-NC 4.0).

(C) Copyright: the Author(s), 2019

Licensee PAGEPress, Italy

Biomedical Science and Engineering 2019; 3(s2):93 doi:10.4081/bse.2019.93

the next future, Janus) is currently used by the UBA to screen large sets of potential PBT chemicals, thus refining and reducing the need of animal testing. Another ongoing project with uba, toDivine, will led to the development of a platform for the eco-toxicological assessment of single compounds, combining the use of QSARs with a readacross approach.

\section{Conclusions}

There is a strong need and request for tools that can be used for quick, reliable and nonexpensive screening and assessment of chemicals, in particular for regulatory activities. In the experience of these past years, we have been able to work on several tools that met a positive feedback from users, and that are actually used both by industries and regulatory bodies.

\section{References}

1. Benfenati E, Roncaglioni A, Lombardo A, Manganaro A. Integrating QSAR, Read-Across, and Screening Tools: The VEGAHUB Platform as an Example. Adv Comput Toxicol 2019;365-81.

2. ECHA. How to use and report (Q)SARs. Available from: https://echa.europa. eu/it/practical-guides

3. Pizzo F, Lombardo A, Manganaro A, et al. Integrated in silico strategy for PBT assessment and prioritization under REACH. Environ Res 2016;151:47892. 